\title{
Revealing Electronic, Structural and Magnetic Phases in NdFeAsO with Electron Energy-Loss Spectroscopy
}

J.C. Idrobo, ${ }^{* * *}$ M.F. Chisholm, ${ }^{* *}$ M. Prange,,${ }^{* *}$ J. Tao, ${ }^{* * *}$ Y. Zhu, ${ }^{* *}$ Z.-A. Ren, ${ }^{* * * *}$ Z.X. Zhao $^{* * * * *}$, S.T. Pantelides, ${ }^{* * *}$ and S.J. Pennycook ${ }^{* * * *}$

* Department of Physics and Astronomy, Vanderbilt University, Nashville, TN 37235

** Materials Science and Technology Division, Oak Ridge National Laboratory,Oak Ridge, TN 37831.

*** Brookhaven National Laboratory, Upton, NY 11973.

${ }^{* * * *}$ Institute of Physics and Beijing National Laboratory for Condensed Matter Physics, Chinese Academy of Sciences, P. O. Box 603, Beijing 100190, P. R. China.

The new iron-based family of high-Tc superconductors has generated interest in the scientific community because these materials present hybrid properties from both classical BCS and highTc superconductors [1]. Iron pnictides, quaternary compounds of the form $(\mathrm{ReFeAsO}, \mathrm{Re}=$ rareearth elements), present structural and magnetic phase transitions in the temperature range between $120 \mathrm{~K}$ to $150 \mathrm{~K}$. Here, we report momentum transfer-dependent and temperaturedependent electron energy-loss spectroscopy (EELS) studies of the parent compound $\mathrm{NdFeAsO}$ using (scanning) transmission electron microscopy and density functional theory.

Z-contrast images were taken at $300 \mathrm{kV}$ in a FEI Titan 80-300 S scanning transmission electron microscope (STEM), equipped with a CEOS probe aberration corrector, and in a dedicated STEM NION UltraSTEM at $100 \mathrm{kV}$, equipped with a NION probe aberration corrector. Both microscopes are located in Oak Ridge National Laboratory. The EEL spectra were acquired in the NION STEM microscope and in a JEOL 3000F operated at $300 \mathrm{kV}$, located in Brookhaven National Laboratory.

We find that both the $\mathrm{Fe} \mathrm{L}_{23}$-ratio and the $\mathrm{Nd} \mathrm{M}_{45}$-ratio change for different crystallographic orientations (Fig. 1). Additionally, the Fe L23-ratio and the $\mathrm{Nd} \mathrm{M} \mathrm{M}_{45}$-ratio increase, while the intensities of the $\mathrm{Fe} \mathrm{L}_{1}$-edge and $\mathrm{Nd} \mathrm{M}_{3}$-edge reduce as the temperature decreases (Fig. 2). Using a combination of experiments and total-energy first-principles calculations within density functional theory, we will show that the changes of the EELS Fe and $\mathrm{Nd}$ fine structure can be directly correlated with changes on the electronic structure of $\mathrm{NdFeAsO}$ and $\mathrm{Fe}$ and $\mathrm{Nd}$ magnetic moments [2].

\section{References:}

[1] Y. Kamihara, et al. J. Am. Che. Soc, 128 (2006) 10012.

[2] This research was supported by the National Science Foundation under Grant No. DMR-0938330 (JCI), the Office of Basic Energy Sciences, Division of Materials Sciences and Engineering (MFC \& SJP), Grant No. DE-FG02-09ER46554 (MP \& STP), the SHaRE User Facility (JCI), which is sponsored by the Division of Scientific User Facilities, Office of Basic Energy Sciences, U.S. Department of Energy. 

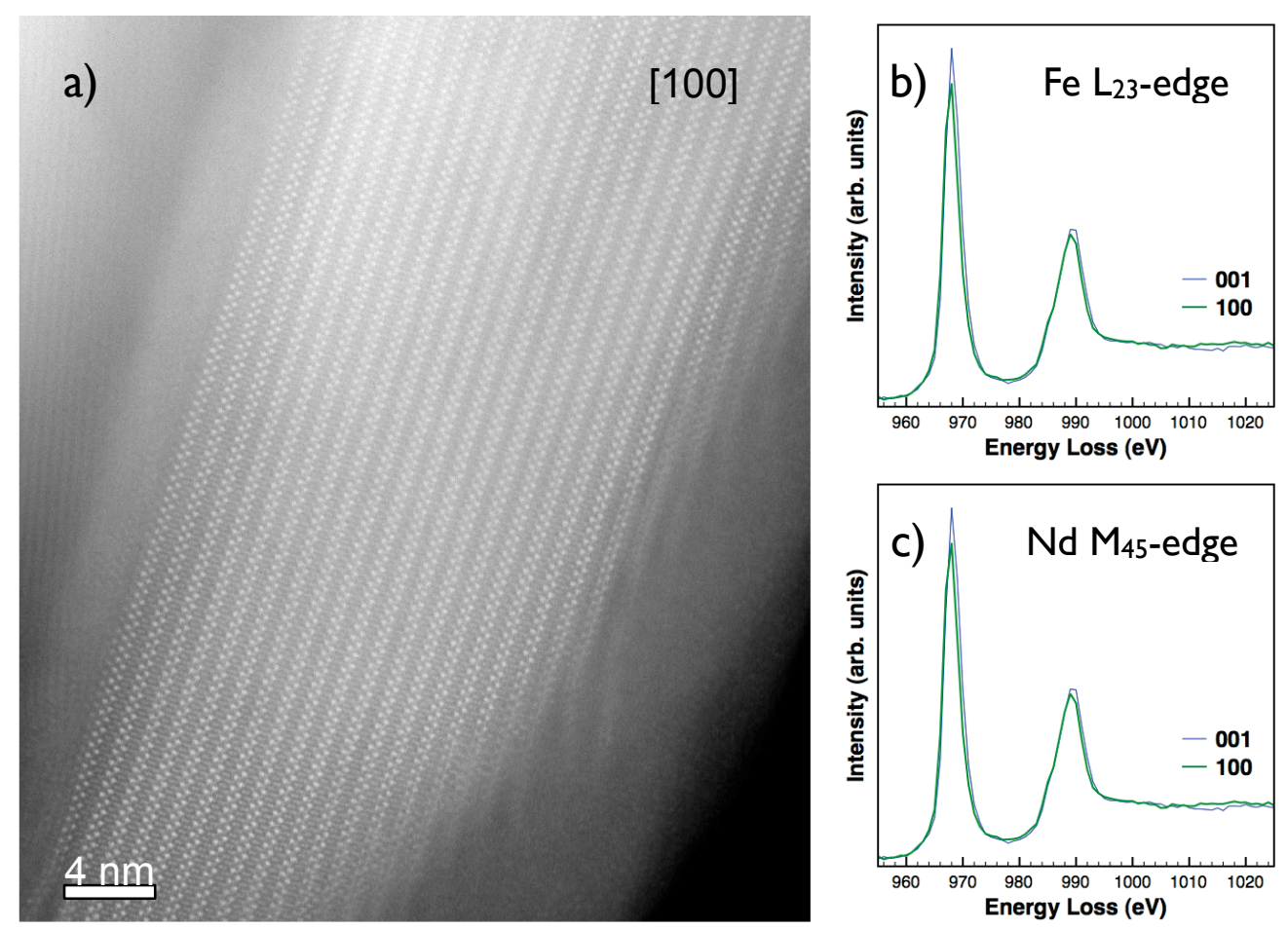

FIG. 1. (a) Z-contrast image NdFeAsO grain along the [001] crystallographic orientation. EEL spectra of (b) Fe L23-edge and (c) Nd M45-edge acquired in the 001 and 100 crystallographic orientations. Image obtained in the FEI Titan 80-300 S at $300 \mathrm{kV}$. Spectra collected in the NION UltraSTEM at $100 \mathrm{kV}$.
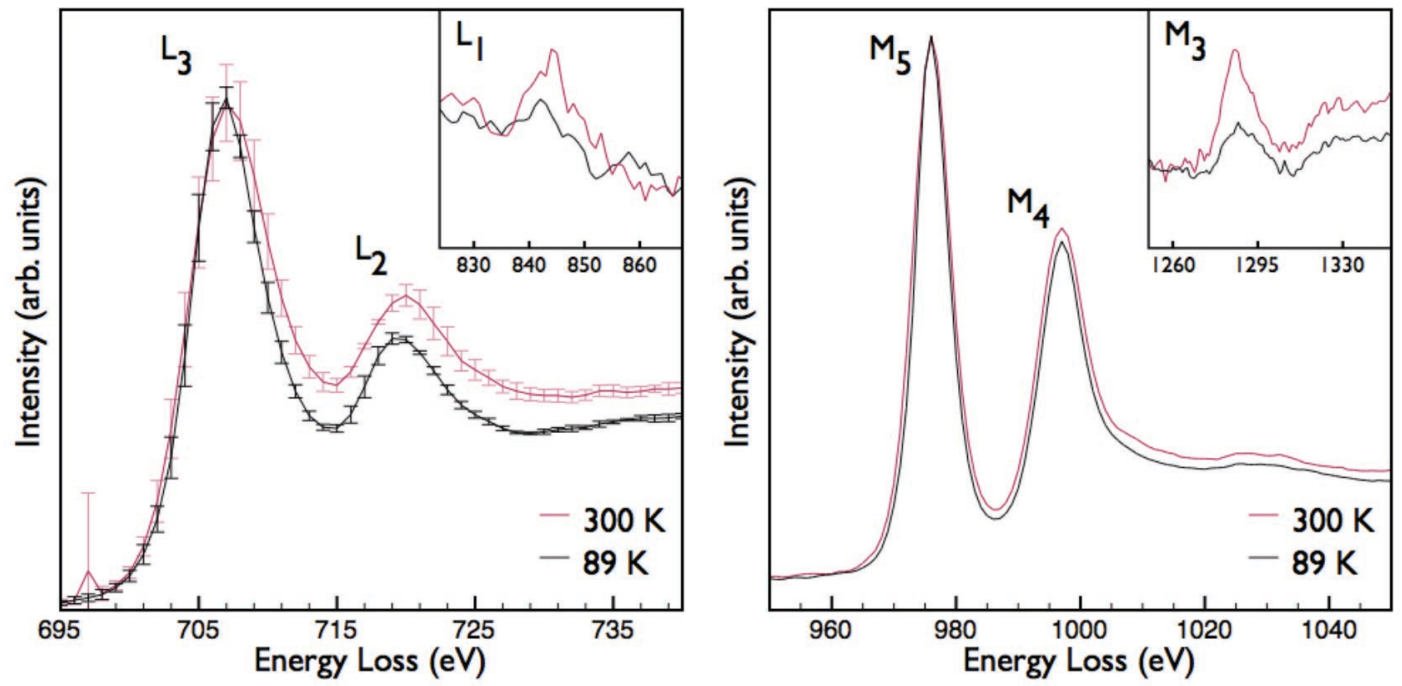

FIG. 2. Iron L-edge and $\mathrm{Nd} \mathrm{M-edge} \mathrm{spectra} \mathrm{taken} \mathrm{from} \mathrm{a} \mathrm{NdFeAsO}$ grain along the [001] crystallographic orientation. The spectra was taken at two different temperatures, $89 \mathrm{~K}$ and 300 $\mathrm{K}$. Both $\mathrm{Fe} \mathrm{L}_{23}$ ratio and $\mathrm{Nd} \mathrm{M}_{45}$ ratios and the intensities of the $\mathrm{Fe}_{1} \mathrm{~L}_{1}$-edge and $\mathrm{Nd} \mathrm{M}_{3}$-edge reduce as the temperature decrease. Spectra collected in TEM mode in the JEOL 3000F operated at $300 \mathrm{kV}$ located in Brookhaven National Laboratory. 